\title{
Effect of Wildfire on Herbaceous Vegetation in Cypress Mixed Oak Forest of Nainital, Kumaun Himalaya, India
}

\author{
Rachita Pandey, Vibhuti, Himani Karki, Pankaj Awasthi, Kiran Bargali, Surendra Singh Bargali* \\ Department of Botany, Kumaun University, Uttarakhand, India
}

"Corresponding author: Surendra Singh Bargali, Department of Botany, DSB Campus, Kumaun University, Nainital-263002(Uttarakhand), India. Tel: +919410579117; Email: surendrakiran@rediffmail.com

Citation: Pandey R, Vibhuti, Karki H, Awasthi P, Bargali K, et al. (2018) Effect of Wildfire on Herbaceous Vegetation in Cypress Mixed Oak Forest of Nainital, Kumaun Himalaya, India. Curr Trends Forest Res: CTFR-121. DOI: 10.29011/2638-0013.100021

Received Date: 21 August, 2018; Accepted Date: 30 August, 2018; Published Date: 07 September, 2018

\begin{abstract}
Forest fire has diverse impacts on atmospheric chemistry, biogeochemical cycling and ecosystem structure and has adverse ecological, economic and social implications. Besides directly damaging the forest trees, the fire also adversely affects forest regeneration, microclimate, soil erosion, and wild life. In most of the cases, the forest fire causes retrogression of forest vegetation. Present study examined the effect of wildfire on herbaceous vegetation in cypress mixed oak forest of Nainital, Kumaun Himalaya, India. The study site is present at and around Aryabhatta Research Institute of Observational Science in Nainital district and three sites (viz., hill base $[1750 \mathrm{~m}]$ hill slope $[1800 \mathrm{~m}]$ and hill top $[1850 \mathrm{~m}]$ ) were selected. The results revealed that a total of 36 herb species representing 18 families were identified, Asteraceae ( 7 species) being the most rich family with maximum number of herb species. Total herb density was $19.60 \mathrm{ind} . \mathrm{m}^{-2}$ at hill base, $21.8 \mathrm{ind} . \mathrm{m}^{-2}$ at hill slope and 33.7 ind. $\mathrm{m}^{-2}$ at hill top. The total herb biomass was $212.57 \mathrm{gm}^{-2}$ at hill base, $116.22 \mathrm{gm}^{-2}$ at hill slope and $141.97 \mathrm{gm}^{-2}$ at hill top. The species composition and richness were significantly affected by burning, the magnitude of effect was generally small. Overall the differences were observed in species composition after fire as a larger group of perennial herbs, primarily grasses and summer forbs, exhibited the changes in density and biomass and overall biodiversity decreased after fire.
\end{abstract}

Keywords: Biomass; Density; Herb; Indian Himalaya; Wildfire

\section{Introduction}

The Central Himalaya accounts for $8.68 \%$ of the total Indian Himalayan region $\left(59,436 \mathrm{~km}^{2}\right)$ and harbors rich biodiversity due to geographical and geological peculiarities subtending a wide range of vegetation types [1]. The Himalaya is recognized for its ecosystem services to the Asian region as well as to the world at large for maintaining slope stability, regulating hydrological integrity, sustaining high levels of biodiversity and human wellbeing. The Himalayan biodiversity is severely threatened by natural (catastrophic wind, landslides) and anthropogenic means (overgrazing, overlopping, habitat fragmentation due to overpopulation). The various disturbances present in the area are eroding this rich biological diversity day by day and has led to the expansion of xerophytic conditions [2]. One of the most prevalent causes of global deforestation and destruction of wildlife is fire. Forest fire is one of the major disasters in the forests of Uttarakhand that adversely affect many indigenous and endangered species every year. Forest fires spread at different speeds depending on vegetation, weather conditions, and physical features.

Quercus is possibly the greatest natural ecosystem-forming genus of the world, it serves as a 'keystone' species in the region because of its significant contribution in soil and water conservation and to sustain rural ecosystems [3]. The major forest forming species of the moist temperate forest of the Indian Himalayan region are Oaks (Quercus spp.) [3,4] and Cypress (Cupressus torulosa) [5]. The genus Quercus (oaks) belong to the family Fagaceae. Oaks are one of the most important groups of flowering plants and dominate large regions of the Northern Hemisphere [4]. Quercus includes about 35 species distributed between 1000-3500 m elevations in Indian Himalayan region. Among the 35 species of Oaks, 5 species are evergreen and grow naturally in the Uttarakhand state.

Associated with these oak woodlands is Cupressus torulosa D. Don, commonly known as Himalayan cypress which is an evergreen tree in the family Cupressaceae. This species is distributed throughout the Himalayan region at elevation between 1800 to $2800 \mathrm{~m}$ [6]. It is indigenous to Western Himalaya and distributed throughout the outer and middle ranges of Himalaya 
from Chamba (HP) to the Nepal $[7,8]$. These forests are of immense significance from the environmental conservation and sustainable development view point as they provide a diverse range of resources and contribute significantly in carbon sequestration and water purification [9].

The herbaceous layer, also referred to as understory or ground layer vegetation is generally defined as all plants (woody or herbaceous) $<1 \mathrm{~m}$ in height, though taller woody vegetation may be included $[10,11]$. Plants in the understorey layer, maintain the structure and functioning of forests $[2,4,12-14]$. It is considered that oak and mixed oak forests support the herbaceous diversity $[3,4]$. Across oak forest landscapes, the herbaceous layer harbors great majority of plant diversity, including most rare plant species. In diverse landscapes and significant topographic heterogeneity, herb layer composition and diversity vary along with aspect related gradients of microclimate, soil moisture and fertility [15]. However, increasing anthropogenic pressures such as population explosion, overgrazing, land degradation for agricultural purposes within Himalayan zone from some decades has caused extensive deforestation, massive soil erosion in the hills, floods in the plains and consequently the silting of rivers [16].

Herb layer vegetation is also affected by natural and anthropogenic disturbances to the tree canopy, individual tree falls, catastrophic wind events and timber harvesting, which result in large increase in resource availability $[11,17,18]$.

Fire is a common feature in Indian Himalayan forests every year, causing incalculable damage to the forest wealth and ecosystem. Any fire on a forestland which was not being used as a tool in forest protection and management and is not in accordance with an authorized plan may be referred to as a wildfire $[18,19]$. Primary causes of wildfires may be natural including lightening or volcanic eruption or human induced including smoking, unattended campfires, burning debris and fireworks. Surface fires usually cause minor damage to overstory trees but affect herb layer vegetation directly by killing aboveground stems and indirectly by altering the forest floor and the availability of light, water and nutrients $[20,21]$. Spatial and temporal variation in severity within a fire can have long-lasting impacts on the structure and species composition of post-fire communities and the potential for future disturbances [22]. Several studies showed variable effects of fire on herb layervegetation in oak forests [23-26].

In India, normal fire season extends from February to mid-June, early summer months are most prone to human induced fire. Frequently, many fires are caused during the burning of crop remains in the agricultural fields that are located near forest or due to a live cigarette or bidi butt thrown on the ground, even due to cooking and campfire activities of local people or tourists. Sometimes the local people deliberately set fire in Chir Pine (Pinus roxburghii) forests to promote growth of understory herbaceous vegetation which can be used as fodder during the monsoon season. In the summer of 2015, there was a severe fire in Uttarakhand Himalaya including the study area so an attempt has been made to highlight the effects of fire as an agent of transformation which affects biotic and abiotic component of natural ecosystem and thus altering productive, protective functions and services of forest ecosystems. The present paper is an attempt to analyze the effect of wildfire on herbaceous vegetation in cypress mixed oak forests of Nainital, Kumaun Himalaya, India.

\section{Materials and Methods Study Site}

The study area is located near the town of Nainital $\left(29^{\circ} 23^{\prime} \mathrm{N}\right.$ $-79^{\circ} 27^{\prime} \mathrm{E}, 29.38^{\circ} \mathrm{N}-79.45^{\circ} \mathrm{E}$ ) at an altitude of 1,950 meters above sea level in Kumaun Himalaya. The study site was present at and around Aryabhatta Research Institute of Observational Sciences (ARIES) (1900 m) in Nainital district and three sites (viz., hill base $[1750 \mathrm{~m}]$ hill slope $[1800 \mathrm{~m}]$ and hill top $[1850 \mathrm{~m}]$ were selected (Photo plate 1). During 2015, in summers there was a heavy fire in Uttarakhand Himalaya including the study area. 


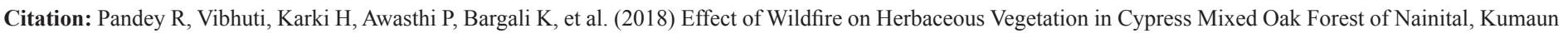
Himalaya, India. Curr Trends Forest Res: CTFR-121. DOI: 10.29011/2638-0013. 100021

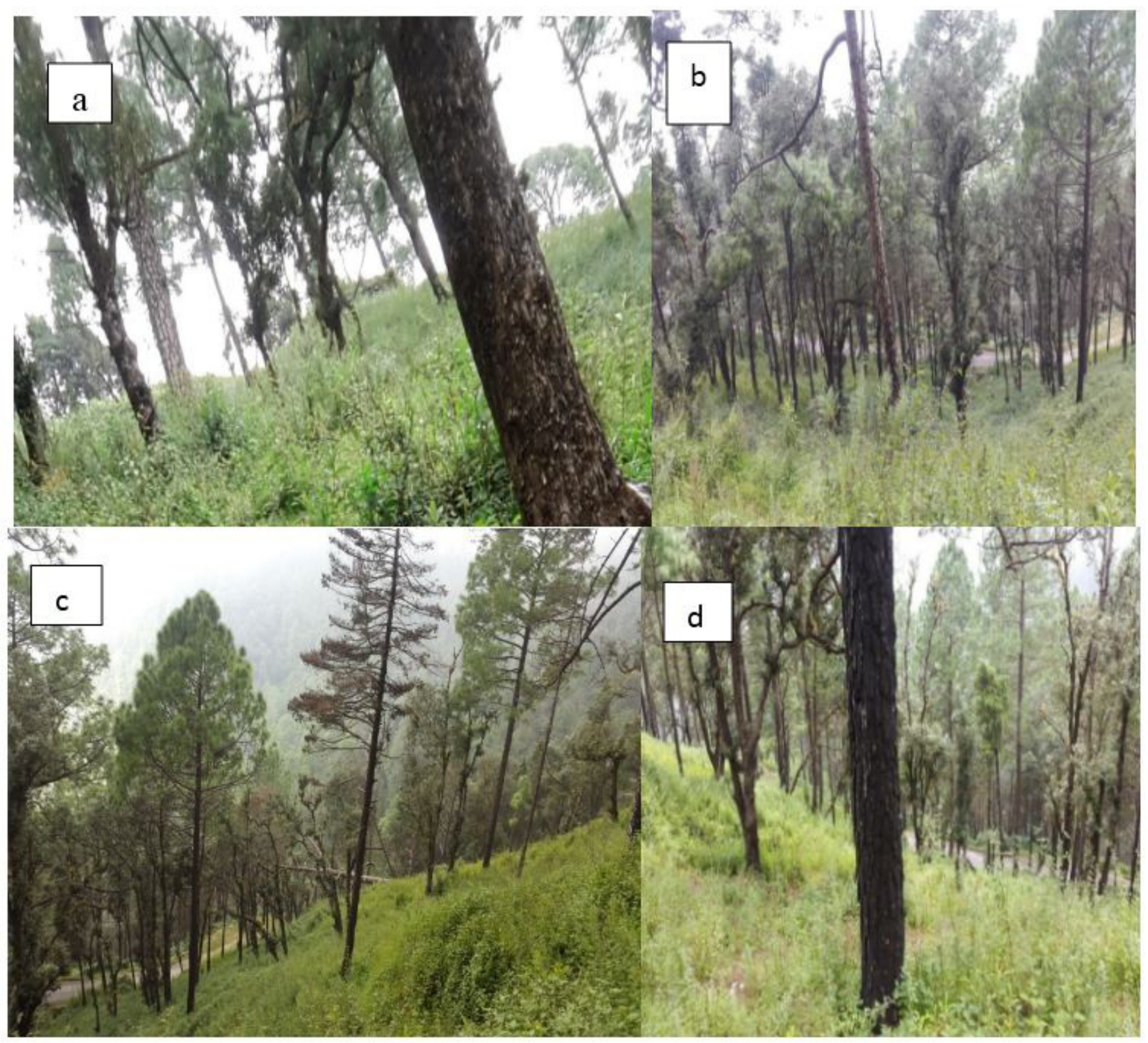

Photo plate 1: Photographs of the study sites (a-Hill top, b-Hill base, c-Hill slope, d- Image depicting severely burnt tree due to fire) in Uttarakhand state (India).

\section{Climate}

The climate of Nainital is classified as Koppen climate type, which is characterized by long-cold often snowy winter and short summer. It is temperate and monsoon type (Singh and Singh, 1992) and the year have four distinct seasons viz., monsoon (July to September), post-monsoon (October to November), winter
(December to January) and summer (April to mid-June). Climatic data for the year 2016 was obtained from the State Observatory at Nainital. The mean annual rainfall was recorded $280.72 \mathrm{~mm}$ and mean monthly rainfall ranged between $0 \mathrm{~mm}$ (November) to $1076.34 \mathrm{~mm}$ (July). The minimum temperature ranged from $6^{\circ} \mathrm{C}$ (January) to $25^{\circ} \mathrm{C}$ (June) and the mean maximum day temperature 
varied from $22^{\circ} \mathrm{C}$ (December) to $39^{\circ} \mathrm{C}$ (May) (Source: ARIES, Nainital) (Figure 1).

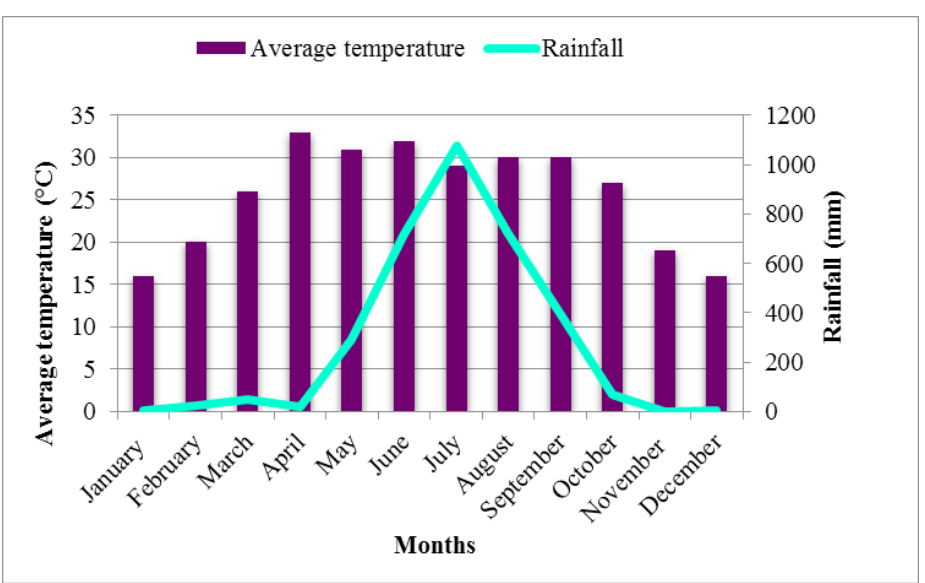

Figure 1: Metrological data of the study site for the year 2016 (Source: ARIES, Nainital).

\section{Sampling}

Plant species were collected carefully from the selected three sites (hill top, hill slope, hill base) and the collected specimens were properly spread in between the newspaper sheets for drying. The dried specimens were then mounted on the herbarium sheets of $29.2 \times 41.9 \mathrm{~cm}$. The collected plant material was identified by consulting the representative flora [27-30] and with the help of Prof. P.C. Pande, Taxonomist, Department of Botany, DSB Campus, Nainital. The herbarium was prepared by standard methods.

The vegetational analysis of herb species was conducted by doing stratified random sampling based position along a slope gradient by placing 10 quadrats of $1 \mathrm{~m} \times 1 \mathrm{~m}$ size randomly at different locations on each of the three sites (viz., hill base, hill slope and hill top). The size and number of samples were determined following Saxena and Singh [31]. Grasses were sampled through tiller analysis and each tiller of grass was considered as an individual plant and creeping plants were counted on the basis of presence of functional roots. The vegetational data were quantitatively analyzed for abundance, density and frequency [32]. The Provenance value $(\mathrm{PV})$ of herbs was determined as the sum of the relative frequency and relative density [33]. The ratio of abundance to frequency indicates distribution pattern which may be regular $(\mathrm{A} / \mathrm{F} \leq 0.025)$ or random $(\mathrm{A} / \mathrm{F}=0.025$ to 0.05$)$ or contagious $(\mathrm{A} / \mathrm{F} \geq 0.05)$ [34]. The diversity index $\left(\mathrm{H}^{\prime}\right)$ was computed by using Shannon-Wiener
Index [35]. The concentration of dominance (CD) was computed by using Simpson's Index [36], species richness was calculated by following Whittaker [37] and equitability was computed by following Pielou [38] and Index of similarity was calculated following Mueller-Dombois and Ellenburg [39]. The statistical analysis was performed using SPSS (version 16.0).

\section{Result}

\section{Composition}

36 herb species, belonging to 36 genera and 18 families were identified (Table 7) and number of herb species ranged from 15 (hill base) to 18 (hill top). The herbs were roughly distributed in a proportion of about $1 / 3^{\text {rd }}$ in each site. From the identified species, pteridophytes were represented by one or two species and the rest of the species were angiosperms. Maximum herb species were contributed by family Asteraceae ( 7 species) followed by Poaceae (5 species) and Lamiaceae (4 species). Arthraxon lanceolatus dominated at hill base and hill slope, while Selaginella spp. dominated at hill top. These results are supported by the fact that higher altitude promotes heterogeneity. Higher number of herb species present at hill top may be due to open canopy of $C$. torulosa due to which more species got chance to establish themselves.

\section{Herb Density and Provenance Value}

Maximum herb density was observed at hill top (33.7 ind. $\left.\mathrm{m}^{-2}\right)$ followed by hill slope (21.8 ind $\left.\mathrm{m}^{-2}\right)$ and hill base (19.60 ind $\left.\mathrm{m}^{-2}\right)$. At hill top, the maximum individual herb density was $11.4 \mathrm{ind} \mathrm{m}^{-2}$ for Selaginella bryopteris and minimum 0.1 ind. $\mathrm{m}^{-2}$ for Cassia spp. Provenance value (PV) indicated the dominance of $S$. bryopteris ( $\mathrm{PV}=43.83)$, predominance of Craniotome versicolor $(\mathrm{PV}=20.51)$ and co-dominance of Parietaria officinalis ( $\mathrm{PV}=18.73$ ). At hill slope, the maximum individual herb density was 8 ind. $\mathrm{m}^{-2}$ for $S$. bryopteris and minimum 0.2 ind. $\mathrm{m}^{-2}$ for Dicliptera bupleuroides and Clematis buchaniana at hill base. Provenance value (PV) indicated the dominance of Arthraxon lanceolatus $(\mathrm{PV}=56.43)$ predominance of $S$. bryopteris $(\mathrm{PV}=47.51)$ and co-dominance of Ainsliaea aptera $(\mathrm{PV}=14.48)$. At hill base, maximum individual herb density was 9.30 ind. $\mathrm{m}^{-2}$ for $A$. lanceolatus and 0.10 ind. $\mathrm{m}^{-2}$ for Salvia officinalis, Cardamine amara, Bidens alba, Fragaria vesca, Cynodon dactylon and Erigeron belloides (Table 1). In the present study, two types of distributional patterns, viz. random and contagious were observed. Random distribution was rare whereas, contagious distribution was common. 


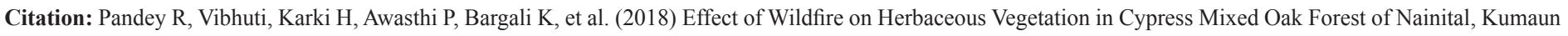
Himalaya, India. Curr Trends Forest Res: CTFR-121. DOI: 10.29011/2638-0013. 100021

\begin{tabular}{|c|c|c|c|c|c|c|c|}
\hline Species & $\begin{array}{l}\text { Density (D) } \\
\text { (ind. } \mathbf{m}^{-2} \text { ) }\end{array}$ & $\begin{array}{l}\text { Frequency } \\
\text { (F) }(\%)\end{array}$ & $\begin{array}{c}\text { Abundance } \\
\text { (A) }\end{array}$ & $\mathbf{A} / \mathbf{F}$ & $\begin{array}{c}\text { Relative } \\
\text { Frequency } \\
(\%)\end{array}$ & $\begin{array}{c}\text { Relative } \\
\text { Density } \\
(\%)\end{array}$ & $\begin{array}{l}\text { Provenance } \\
\text { Value( PV) }\end{array}$ \\
\hline \multicolumn{8}{|c|}{ Hill Base } \\
\hline Salvia officinalis & 0.10 & 10 & 01.00 & 0.10 & 03.13 & 00.51 & 03.64 \\
\hline Dicliptera bupleuroides & 4.40 & 60 & 07.33 & 0.12 & 18.75 & 22.45 & 41.20 \\
\hline Arthraxon lanceolatus & 9.30 & 70 & 13.29 & 0.19 & 21.88 & 47.45 & 69.32 \\
\hline Cardamine amara & 0.10 & 20 & 01.00 & 0.05 & 06.25 & 00.51 & 06.76 \\
\hline Roylea cinerea & 0.60 & 20 & 06.00 & 0.30 & 06.25 & 03.06 & 09.31 \\
\hline Achyranthes bidentata & 0.80 & 30 & 02.67 & 0.09 & 09.38 & 04.08 & 13.46 \\
\hline Bidens alba & 0.10 & 20 & 01.00 & 0.05 & 06.25 & 00.51 & 06.76 \\
\hline Fragaria vesca & 0.10 & 10 & 01.00 & 0.10 & 03.13 & 00.51 & 03.64 \\
\hline Conyza agerotoides & 0.50 & 10 & 05.00 & 0.50 & 03.13 & 02.55 & 05.68 \\
\hline Artemisia annua & 1.00 & 10 & 10.00 & 1.00 & 03.13 & 05.10 & 08.23 \\
\hline Cynodon dactylon & 0.10 & 10 & 01.00 & 0.10 & 03.13 & 00.51 & 03.64 \\
\hline Erigeron bellidiodes & 0.10 & 10 & 01.00 & 0.10 & 03.13 & 00.51 & 03.64 \\
\hline Verbascum thapsus & 0.60 & 10 & 6.00 & 0.60 & 03.13 & 03.06 & 06.19 \\
\hline Seigesbeckia orientalis & 00.80 & 10 & 08.00 & 00.80 & 03.13 & 04.08 & 07.21 \\
\hline Eupatorium adenophorum & 01.00 & 20 & 05.00 & 00.25 & 06.25 & 05.10 & 11.35 \\
\hline Total & 19.60 & & 69.29 & & & & 200 \\
\hline \multicolumn{8}{|c|}{ Hill Slope } \\
\hline Thalictrum foliolosum & 0.5 & 10 & 05 & 0.5 & 2.70 & 2.29 & 5.00 \\
\hline Selaginella bryopteris & 08 & 40 & 20 & 0.5 & 10.81 & 36.70 & 47.51 \\
\hline
\end{tabular}




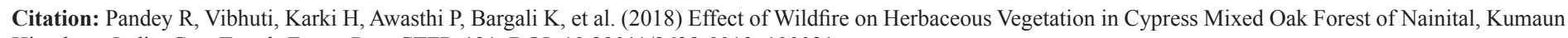
Himalaya, India. Curr Trends Forest Res: CTFR-121. DOI: 10.29011/2638-0013. 100021

\begin{tabular}{|c|c|c|c|c|c|c|c|}
\hline Ainsliaea aptera & 0.8 & 40 & 02 & 0.05 & 10.81 & 3.67 & 14.48 \\
\hline Arthraxon lanceolatus. & 07 & 90 & 7.78 & 0.09 & 24.32 & 32.11 & 56.43 \\
\hline Ageratum conyzoides & 0.9 & 20 & 4.5 & 0.23 & 5.41 & 4.13 & 9.53 \\
\hline Justicia simplex & 01 & 20 & 05 & 0.25 & 5.41 & 4.59 & 9.99 \\
\hline Achyranthes bidentata & 0.5 & 30 & 1.67 & 0.06 & 8.11 & 2.29 & 10.40 \\
\hline Роа аппиа & 0.4 & 10 & 04 & 0.4 & 2.70 & 1.83 & 4.54 \\
\hline Randia densiflora & 0.7 & 40 & 1.75 & 0.04 & 10.81 & 3.21 & 14.02 \\
\hline Dicliptera bupleuroides & 0.2 & 10 & 02 & 0.2 & 2.70 & 0.92 & 3.62 \\
\hline Clematis buchaniana & 0.2 & 10 & 2 & 0.2 & 2.70 & 0.92 & 3.62 \\
\hline Carex hirta & 0.3 & 20 & 1.5 & 0.08 & 5.41 & 1.38 & 6.78 \\
\hline Vitis himalayana & 0.6 & 20 & 3 & 0.15 & 5.41 & 2.75 & 8.16 \\
\hline Strobilanthes callosa & 0.7 & 10 & 7 & 0.7 & 2.70 & 3.21 & 5.91 \\
\hline Total & 21.8 & & & & & & 200 \\
\hline \multicolumn{8}{|c|}{ Hill Top } \\
\hline Arthraxon lanceolatus & 3.6 & 30 & 12.00 & 0.40 & 7.5 & 10.68 & 18.18 \\
\hline Clematis buchaniana & 1.2 & 30 & 4.00 & 0.13 & 7.5 & 3.56 & 11.06 \\
\hline Fragaria vesca & 1.1 & 10 & 11.00 & 1.10 & 2.5 & 3.26 & 5.76 \\
\hline Crysopogon zizanioides & 2.3 & 30 & 7.67 & 0.26 & 7.5 & 6.82 & 14.32 \\
\hline Craniotome versicolor & 2.7 & 50 & 5.40 & 0.11 & 12.5 & 8.01 & 20.51 \\
\hline Eupatorium adenophorum & 1.8 & 20 & 9.00 & 0.45 & 5.0 & 5.34 & 10.34 \\
\hline Micromeria & 1.7 & 30 & 5.67 & 0.19 & 7.5 & 5.04 & 12.54 \\
\hline
\end{tabular}




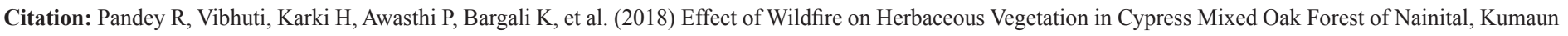
Himalaya, India. Curr Trends Forest Res: CTFR-121. DOI: 10.29011/2638-0013. 100021

\begin{tabular}{|c|c|c|c|c|c|c|c|}
\hline Cynodon dactylon & 0.8 & 20 & 4.00 & 0.20 & 5.0 & 2.37 & 7.37 \\
\hline Artemisia annua & 1.0 & 10 & 10.00 & 1.00 & 2.5 & 2.97 & 5.47 \\
\hline Selaginella bryopteris & 11.4 & 40 & 28.50 & 0.71 & 10 & 33.83 & 43.83 \\
\hline Cyanotis axillaris & 1.1 & 20 & 5.50 & 0.28 & 5.0 & 3.26 & 8.26 \\
\hline Parietaria officinalis & 2.1 & 50 & 4.20 & 0.08 & 12.5 & 6.23 & 18.73 \\
\hline Stellaria media & 0.3 & 10 & 3.00 & 0.30 & 2.5 & 0.89 & 3.39 \\
\hline Paspalum conjugatum & 0.5 & 10 & 5.00 & 0.50 & 2.5 & 1.48 & 3.98 \\
\hline Cassia occidentalis & 0.1 & 10 & 1.00 & 0.10 & 2.5 & 0.30 & 2.80 \\
\hline Gallium aparina & 1.5 & 10 & 15.00 & 1.50 & 2.5 & 4.45 & 6.95 \\
\hline Verbascum thapsus & 0.3 & 10 & 3.00 & 0.30 & 2.5 & 0.89 & 3.39 \\
\hline Cynoglossum officinale & 0.2 & 10 & 2.00 & 0.20 & 2.5 & 0.59 & 3.09 \\
\hline Total & 33.7 & & 135.93 & & & & 200 \\
\hline
\end{tabular}

Table 1: Vegetation analysis of herb layer in the Cypress mixed oak forest.

On the basis of percent similarity, Hill base and Hill slope were $20.68 \%$ similar, the Hill slope and Hill top showed $18.75 \%$ similarity and the Hill Top and Hill base were $48.48 \%$ similar in herb layer which showed maximum similarity (Table 2). Across the three sites, maximum diversity was observed in hill slope for S. bryopteris $(0.53)$ and minimum in hill top for Cassia occidentalis $(0.02)$. Maximum concentration of dominance was observed for A. lanceolatus $(0.22)$ in hill base. Equitability was observed maximum for $S$. bryopteris and A. lanceolatus (0.20) in hill slope. (Table 3)

\begin{tabular}{|c|c|c|}
\hline Sites & Hill Slope & Hill Top \\
\hline Hill Base & $20.68 \%$ & $48.48 \%$ \\
\hline Hill Slope & - & $18.75 \%$ \\
\hline
\end{tabular}

Table 2: Index of similarity.

\begin{tabular}{|c|c|c|c|c|}
\hline Species & Diversity index (H') & $\begin{array}{c}\text { Concentration of dominance } \\
\text { (Cd) }\end{array}$ & Equitability (e) \\
\hline \multicolumn{5}{|c|}{ Hill base } \\
\hline Salvia officinalis & 0.04 & 0.00 & 0.01 \\
\hline Dicliptera bupleuroides & 0.48 & 0.05 & 0.18 \\
\hline Arthraxon lanceolatus & 0.51 & 0.22 & 0.19 \\
\hline Cardamine amara & 0.04 & 0.00 & 0.01 \\
\hline
\end{tabular}




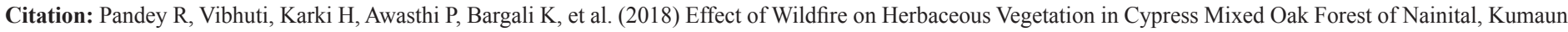
Himalaya, India. Curr Trends Forest Res: CTFR-121. DOI: 10.29011/2638-0013. 100021

\begin{tabular}{|c|c|c|c|}
\hline Roylea cinerea & 0.15 & 0.00 & 0.06 \\
\hline Achyranthes bidentata & 0.19 & 0.00 & 0.07 \\
\hline Bidens alba & 0.04 & 0.00 & 0.01 \\
\hline Fragaria vesca & 0.04 & 0.00 & 0.01 \\
\hline Conyza agerotoides & 0.13 & 0.00 & 0.05 \\
\hline Artemisia annua & 0.22 & 0.00 & 0.08 \\
\hline Cynodon dactylon & 0.04 & 0.00 & 0.01 \\
\hline Erigeron bellidiodes & 0.04 & 0.00 & 0.01 \\
\hline Verbascum thapsus & 0.15 & 0.00 & 0.06 \\
\hline Seigesbeckia orientalis & 0.19 & 0.00 & 0.07 \\
\hline Eupatorium adenophorum & 0.22 & 0.00 & 0.08 \\
\hline \multicolumn{4}{|c|}{ Hill slope } \\
\hline Thalictrum foliolosum & 0.12 & 0.00 & 0.05 \\
\hline Selaginella bryopteris & 0.53 & 0.13 & 0.20 \\
\hline Ainsliaea aptera & 0.17 & 0.00 & 0.07 \\
\hline Arthraxon lanceolatus. & 0.53 & 0.10 & 0.20 \\
\hline Ageratum conyzoides & 0.19 & 0.00 & 0.07 \\
\hline Justicia simplex & 0.20 & 0.00 & 0.08 \\
\hline Achyranthes bidentata & 0.12 & 0.00 & 0.05 \\
\hline Poа апnиа & 0.11 & 0.00 & 0.04 \\
\hline Randia densiflora & 0.16 & 0.00 & 0.06 \\
\hline Dicliptera bupleuroides & 0.06 & 0.00 & 0.02 \\
\hline Clematis buchaniana & 0.06 & 0.00 & 0.02 \\
\hline Carex hirta & 0.09 & 0.00 & 0.03 \\
\hline Vitis himalayana & 0.14 & 0.00 & 0.05 \\
\hline Strobilanthes callosa & 0.16 & 0.00 & 0.06 \\
\hline \multicolumn{4}{|c|}{ Hill top } \\
\hline Arthraxon lanceolatus. & 0.34 & 0.01 & 0.12 \\
\hline Clematis buchaniana & 0.17 & 0.00 & 0.06 \\
\hline Fragaria vesca & 0.16 & 0.00 & 0.06 \\
\hline Crysopogon zizanioide & 0.26 & 0.00 & 0.09 \\
\hline Craniotome versicolor. & 0.29 & 0.01 & 0.10 \\
\hline Eupatorium adenophorum & 0.23 & 0.00 & 0.08 \\
\hline Micromeria biflora & 0.22 & 0.00 & 0.08 \\
\hline
\end{tabular}




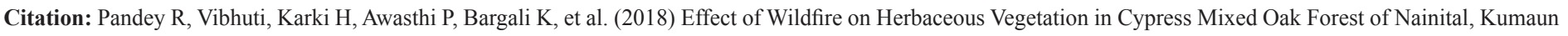
Himalaya, India. Curr Trends Forest Res: CTFR-121. DOI: 10.29011/2638-0013. 100021

\begin{tabular}{|c|l|l|c|}
\hline Cynodon dactylon & 0.13 & 0.00 & 0.04 \\
\hline Artemisia annua & 0.15 & 0.00 & 0.05 \\
\hline Selaginella bryopteris & 0.53 & 0.11 & 0.18 \\
\hline Cyanotis axillaris & 0.16 & 0.00 & 0.06 \\
\hline Parietaria officinalis & 0.25 & 0.00 & 0.09 \\
\hline Stellaria media & 0.06 & 0.00 & 0.02 \\
\hline Paspalum conjugatum & 0.09 & 0.00 & 0.03 \\
\hline Cassia occidentalis & 0.02 & 0.00 & 0.01 \\
\hline Gallium aparina & 0.20 & 0.00 & 0.07 \\
\hline Verbascum thapsus & 0.06 & 0.00 & 0.02 \\
\hline Cynoglossum officinale & 0.04 & 0.00 & 0.02 \\
\hline
\end{tabular}

Table 3: Diversity (H'), Concentration of dominance (Cd) and Equitability (e) of herbs in Cypress Oak mixed forest.

\section{Herb Biomass}

Maximum herb biomass (212.57 $\mathrm{gm}^{-2}$ ) was observed at hill base, whereas minimum biomass was reported at hill slope (116.22 $\mathrm{gm}^{-2}$ ) and the total biomass at hill top $\left(141.97 \mathrm{gm}^{-2}\right)$ ranged in between hill base and hill slope. At hill base, maximum biomass was contributed by D. bupleuroides $(25.83 \%)$ and minimum biomass was contributed by B. alba $(0.10 \%)$. At hill slope, A. lanceolatus contributed highest biomass $(24.88 \%)$ and lowest contribution was reported by P. annua (0.30\%). At hill top, C. zizaniodes contributed highest biomass $(20.57 \%)$ and C. offcinale $(0.77 \%)$ contributed lowest biomass (Table 4$)$.

\begin{tabular}{|c|c|c|c|}
\hline Herb species & $\operatorname{AGB}\left(\mathrm{gm}^{-2}\right)$ & $\mathrm{BGB}\left(\mathrm{gm}^{-2}\right)$ & Total \\
\hline \multicolumn{4}{|c|}{ Hill base } \\
\hline Salvia officinalis & 1.01 & 0.97 & 1.98 \\
\hline Dicliptera bupleuroides & 50.34 & 4.57 & 54.91 \\
\hline Arthraxon lanceolatus & 48.59 & 2.07 & 50.67 \\
\hline Cardamine amara & 1.75 & 1.02 & 2.77 \\
\hline Roylea cinerea & 0.38 & 0.30 & 0.68 \\
\hline Achyranthes bidentata & 14.35 & 6.66 & 21.01 \\
\hline Bidens alba & 0.11 & 0.11 & 0.22 \\
\hline Fragaria vesca & 0.11 & 0.12 & 0.23 \\
\hline Conyza agerotoides & 4.28 & 0.29 & 4.57 \\
\hline Artemisia annua & 1.32 & 0.08 & 1.40 \\
\hline Cynodon dactylon & 0.88 & 0.05 & 0.93 \\
\hline Erigeron bellidiodes & 0.35 & 0.02 & 0.37 \\
\hline Verbascum Thapsus & 15.52 & 25.02 & 40.54 \\
\hline Seigesbeckia orientalis & 2.30 & 0.10 & 2.40 \\
\hline Eupatorium adenophorum & 21.19 & 8.70 & 29.89 \\
\hline
\end{tabular}




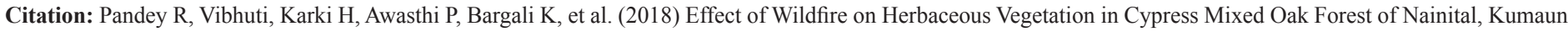
Himalaya, India. Curr Trends Forest Res: CTFR-121. DOI: 10.29011/2638-0013. 100021

\begin{tabular}{|c|c|c|c|}
\hline Total & 162.48 & 50.08 & 212.57 \\
\hline Herb Species & $\operatorname{AGB}\left(\mathrm{gm}^{-2}\right)$ & BGB $\left(\mathrm{gm}^{-2}\right)$ & Total \\
\hline \multicolumn{4}{|c|}{ Hill slope } \\
\hline Thalictrum foliolosum & 3.99 & 1.97 & 5.96 \\
\hline Selaginella bryopteris & 4.08 & 1.22 & 5.30 \\
\hline Ainsliaea aptera & 3.71 & 2.51 & 6.22 \\
\hline Arthraxon lanceolatus. & 24.44 & 4.48 & 28.92 \\
\hline Ageratum conyzoides & 2.35 & 2.25 & 4.60 \\
\hline Justicia simplex & 3.38 & 1.30 & 4.67 \\
\hline Achyranthes bidentata & 2.94 & 3.22 & 6.16 \\
\hline Poа аппиа & 0.31 & 0.05 & 0.36 \\
\hline Randia densiflora & 5.50 & 6.51 & 12.00 \\
\hline Dicliptera bupleuroides & 0.36 & 0.34 & 0.70 \\
\hline Clematis buchaniana & 1.48 & 0.17 & 1.65 \\
\hline Carex hirta & 1.52 & 1.34 & 2.85 \\
\hline Vitis himalayana & 15.84 & 1.10 & 16.94 \\
\hline Strobilanthes callosa & 13.46 & 6.45 & 19.91 \\
\hline Total & 83.35 & 32.87 & 116.22 \\
\hline Herb Species & $\operatorname{AGB}\left(\mathrm{gm}^{-2}\right)$ & $\mathrm{BGB}\left(\mathrm{gm}^{-2}\right)$ & Total \\
\hline \multicolumn{4}{|c|}{ Hill top } \\
\hline Arthraxon lanceolatus. & 11.71 & 0.97 & 12.68 \\
\hline Clematis buchaniana & 3.13 & 1.30 & 4.43 \\
\hline Fragaria vesca & 3.64 & 0.18 & 3.82 \\
\hline Crysopogon zizanioides & 11.76 & 17.45 & 29.21 \\
\hline Craniotome versicolor & 13.12 & 1.79 & 14.91 \\
\hline Eupatorium adenophorum & 12.77 & 2.66 & 15.43 \\
\hline Micromeria biflora & 4.53 & 0.29 & 4.82 \\
\hline Cynodon dactylon & 4.46 & 3.79 & 8.25 \\
\hline Artemisia anпиа & 6.46 & 1.67 & 8.13 \\
\hline Selaginella bryopteris & 9.50 & 1.71 & 11.21 \\
\hline Cyanotis axillaris & 1.75 & 0.75 & 2.50 \\
\hline Parietaria officinalis & 3.31 & 0.69 & 3.99 \\
\hline Stellaria media (L)Vill & 0.39 & 0.90 & 1.29 \\
\hline Paspalum conjugatum & 2.00 & 0.41 & 2.41 \\
\hline
\end{tabular}




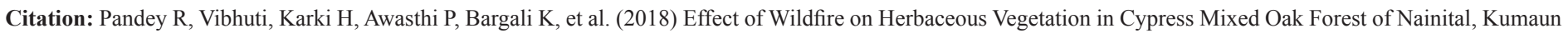
Himalaya, India. Curr Trends Forest Res: CTFR-121. DOI: 10.29011/2638-0013. 100021

\begin{tabular}{|c|c|c|c|}
\hline Cassia occidentalis & 1.98 & 0.33 & 2.31 \\
\hline Gallium aparina & 3.60 & 0.02 & 3.62 \\
\hline Verbascum Thapsus & 10.46 & 1.41 & 11.87 \\
\hline Cynoglossum officinale & 1.03 & 0.07 & 1.10 \\
\hline Total & $\mathbf{1 0 5 . 5 8}$ & $\mathbf{3 6 . 3 8}$ & $\mathbf{1 4 1 . 9 7}$ \\
\hline
\end{tabular}

Table 4: Herb layer biomass in the temperate Cypress dominated mixed-oak forest.

\section{Dominance Diversity Curves}

At hill base, one herb species was clearly dominant, one was predominant and rest of the species showed similar dominance. At hill slope, one herb species was clearly dominant, one was predominant and rest of the species showed similar dominance. At hill top, group of herb species showed similar dominance while one species was clearly dominant (Figure 2).

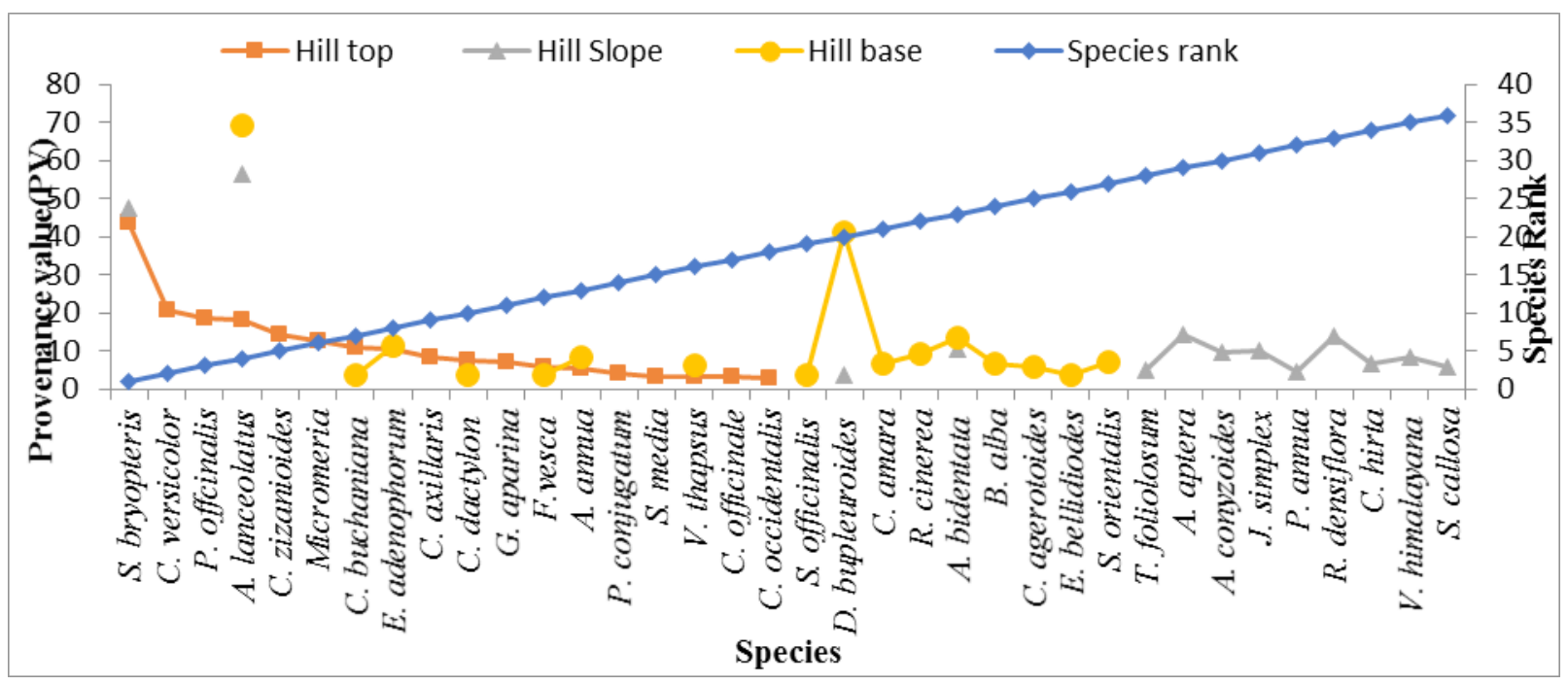

Figure 2: Dominance diversity curve of herb species at Cypress mixed Oak forest.

\section{Discussion}

In the present study a total of 36 herb species, belonging to 36 genera and 18 families were identified (Table 7) while 27 herb species from same site were reported previously by Karki et al. [40] that further supports the fact that the abundance of herbaceous plants increased after fire (Table 7). Hill top occupied highest numbers among the herb species followed by hill base and hill slope. The richest families were Asteraceae $(7$ species) followed by Poaceae ( 5 species), Lamiaceae (4 species $A$. lanceolatus was dominating in hill base and hill slope, while $S$. bryopteris dominated at hill top while 22 to 25 herbs were reported for mixed oak forests of the region [41].

Natural as well as human disturbances influence forest dynamics and plant diversity at local and regional scales [42]. Nearly all studies of fire effects have revealed that the cover and/or abundance of herbaceous plants increased after fire
$[23,25,43,44]$. For woody plant cover, the fire response may be more variable because of the variation in the sprouting response in different vegetation types [44-46]. Fire often has been shown to increase species richness and/or diversity in the herbaceous layer $[25,44,45]$.

Herb-layer vegetation is affected indirectly when fire alters the forest floor and soil environments. Elevated soil temperatures likely affect the germination, establishment and growth of herblayer vegetation. Leaf litter plays an important role in herb-layer plant communities [47]. In the short term, the consumption of leaf litter and humus during fires probably causes the greatest changes in herb-layer vegetation by reducing the mechanical barrier (accumulated litter and debris) and increasing light levels to seeds in the humus and mineral soil. The consumption of leaf litter in fires also releases nutrients that are then incorporated into the mineral soil, altering soil chemistry and likely affecting plant productivity, particularly on nutrient poor sites [48], as fire alters forest structure 


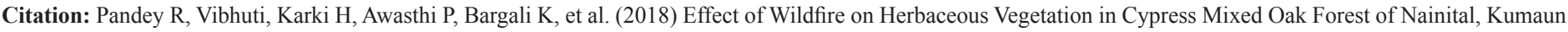
Himalaya, India. Curr Trends Forest Res: CTFR-121. DOI: 10.29011/2638-0013. 100021

by killing of saplings and some trees, light levels increase to the forest floor. Fire also increases the amount of nitrate in soil, this could stimulate the germination of some seed bank [49].

The total density of herbs varied from 19.60 to 33.7 ind. $\mathrm{m}^{-2}$ with hill top having highest density (Table 5). However, herb density ranged from 100.80 to 135.6 ind. $\mathrm{m}^{-2}$ in earlier studies [40]. In the present study site, the total biomass fall between 212.57 $\mathrm{gm}^{-2}$ (at hill base) and 116.22 (at hill slope), while Karki et al. [40] reported the total biomass from $174.71 \mathrm{gm}^{-2}$ to $352.52 \mathrm{gm}^{-2}$ at the same site before fire. Perhaps, the major difference of density and biomass values may be due to increase in anthropological pressures (accidental fire) with development of time.

\begin{tabular}{|c|c|c|}
\hline \multirow[t]{2}{*}{ Herb species } & \multicolumn{2}{|c|}{ Density (ind.m-2) } \\
\hline & $\begin{array}{c}\text { After fire } \\
\text { (Present } \\
\text { study) }\end{array}$ & $\begin{array}{c}\text { Before fire } \\
\text { (Karki et al. } \\
\text { 2016) }\end{array}$ \\
\hline \multicolumn{3}{|c|}{ Hill base } \\
\hline Achyranthes bidentata & 0.8 & 12 \\
\hline Ainsliaea aptera & - & 14.4 \\
\hline Artemisia annua & 1 & 3.2 \\
\hline Arthraxon lanceolatus & 9.3 & 18 \\
\hline Bidens alba & 0.1 & - \\
\hline Cardamine amara & 0.1 & - \\
\hline Clematis buchaniana & - & 0.4 \\
\hline Conyza agerotoides & 0.5 & - \\
\hline Creniotome versicolor & - & 14.8 \\
\hline Cynodon dactylon & 0.1 & 2.4 \\
\hline Dicliptera bupleuroides & 4.4 & - \\
\hline Dioscorea deltoidea & - & 1.6 \\
\hline Erigeron bellidiodes & 0.1 & 44.4 \\
\hline Eupatorium adenophorum & 1 & 9.6 \\
\hline Fragaria vesca & 0.1 & 0.8 \\
\hline Galium aparina & - & 1.6 \\
\hline Goldfussia dalhousianus & - & 1.6 \\
\hline Melaxis acuminate & - & 0.4 \\
\hline Roylea cinerea & 0.6 & - \\
\hline Salvia officinalis & 0.1 & - \\
\hline Seigesbeckia orientalis & 0.8 & - \\
\hline
\end{tabular}

\begin{tabular}{|c|c|c|}
\hline Selaginella spp. & - & 11.2 \\
\hline Verbascum Thapsus & 0.6 & - \\
\hline Viola canescens & - & 2.8 \\
\hline Vitis Himalayana & - & 3.2 \\
\hline \multicolumn{3}{|c|}{ Hill slope } \\
\hline Achyranthes bidentata & 0.5 & 9.2 \\
\hline Ageratum conyzoides & 0.9 & - \\
\hline Ainsliaea aptera & 0.8 & 8.4 \\
\hline Arthraxon lanceolatus. & 7 & 24 \\
\hline Carex hirta & 0.3 & - \\
\hline Clematis buchaniana & 0.2 & - \\
\hline Dicliptera bupleuroides & 0.2 & 13.6 \\
\hline Justicia simplex & 1 & - \\
\hline Poa аппиа & 0.4 & - \\
\hline Randia densiflora & 0.7 & - \\
\hline Selaginella spp. & 8 & 27.6 \\
\hline Strobilanthes callosa & 0.7 & - \\
\hline Thalictrum foliolosum & 0.5 & - \\
\hline Vitis himalayana & 0.6 & 0.8 \\
\hline Cypress rotundus & - & 45.6 \\
\hline Creniotome versicolor & - & 14.8 \\
\hline Stellaria media & - & 2.4 \\
\hline Galium rotundifolium & - & 10 \\
\hline C. dactylon & - & 4 \\
\hline G. dalhousianus & - & 2.8 \\
\hline G. gossypina & - & 2.8 \\
\hline Onychium cryptogrammoides & - & 8 \\
\hline \multicolumn{3}{|c|}{ Hill Top } \\
\hline A. aptera & - & 17.2 \\
\hline A. bidentata & - & 8 \\
\hline Artemisia аппиа & 1 & - \\
\hline Arthraxon lanceolatus & 3.6 & 24.8 \\
\hline C. rotundus & - & 12 \\
\hline Cassia occidentalis & 0.1 & - \\
\hline
\end{tabular}




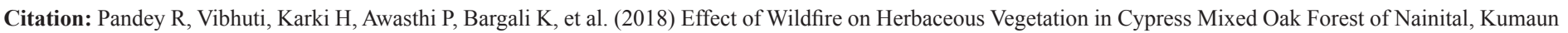
Himalaya, India. Curr Trends Forest Res: CTFR-121. DOI: 10.29011/2638-0013. 100021

\begin{tabular}{|c|c|c|}
\hline Clematis buchaniana & 1.2 & 0.4 \\
\hline Craniotome versicolor & 2.7 & - \\
\hline Crysopogon zizanioides & 2.3 & - \\
\hline Cyanotis axillaris & 1.1 & - \\
\hline Cynodon dactylon & 0.8 & - \\
\hline Cynoglossum officinale & 0.2 & - \\
\hline D. bupleuroids & - & 8.4 \\
\hline Eupatorium adenophorum & 1.8 & - \\
\hline Fragaria vesca & 1.1 & - \\
\hline G. dalhousianus & - & 17.2 \\
\hline G. gossypina & - & 3.2 \\
\hline G. rotundifolium & - & 4.4 \\
\hline Gallium aparina & 1.5 & - \\
\hline Hedychium spicatum & - & 1.2 \\
\hline Micromeria & 1.7 & - \\
\hline Parietaria officinalis & 2.1 & - \\
\hline Paspalum conjugatum & 0.5 & - \\
\hline Selaginella spp. & 11.4 & 62 \\
\hline Stellaria media & 0.3 & - \\
\hline Thalictrum foliolosum & - & 0.8 \\
\hline Tragopogon gracilis & - & 2.8 \\
\hline V. himalyana & - & 2.0 \\
\hline Verbascum Thapsus & 0.3 & - \\
\hline Viola serpens & - & 0.4 \\
\hline
\end{tabular}

$20.68 \%$ similar, the Hill slope and Hill top were $18.75 \%$ similar and the maximum similarity existed between Hill Top and Hill base $(48.48 \%)$. However, earlier studies [40] revealed that Hill slope and Hill top showed $68.97 \%$ similarity, hill base and hill slope showed $51.61 \%$ similarity and hill base and hill top showed $43.75 \%$ similarity (Table 2 ).

\section{Cluster Analysis}

Cluster analysis is a way of grouping cases of data. In the present study, 2 groups were formed on the basis of different parameters (Density, Diversity and Biomass) before and after fire. Across the sub-sites (Hill base, Hill slope, Hill top), group 1, 2 and 3 (before fire) join together to form a cluster with homogenous combination followed by group 4, 5 and 6 group (after fire) which showed heterogeneous combination with group 1,2 and 3 . On the basis of different parameters, group 1, 2, 3 form a single cluster that means hill base, hill slope and hill top of before fire showed similarity whereas group 4, 5, 6 also form a single cluster i.e. hill base, hill slope, hill top after fire also showed similarity but as the clusters formed before fire and after fire showed a wide gap in between i.e. they were strongly dissimilar with respect to the parameters studied (Figure 3).

Dendrogram using Average Linkage (Between Groups)

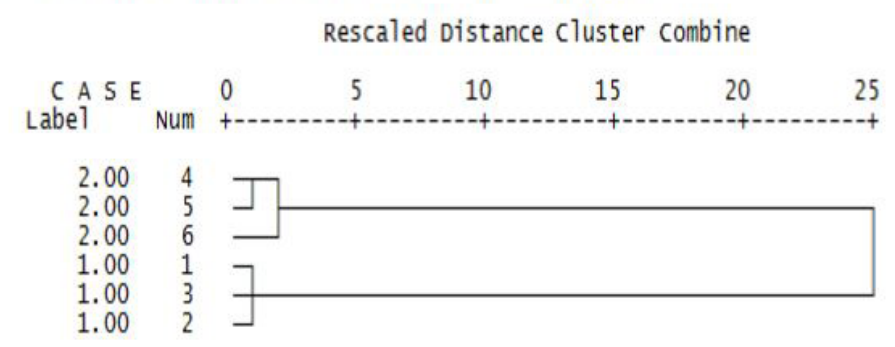

Figure 3: Cluster analysis for different parameters

$1=$ Hill base $\quad 2=$ Hill slope $\quad 3=$ Hill top $(1.00=$ Before fire $)$

4=Hill base $\quad 5=$ Hill slope $\quad 6=$ Hill top $(2.00=$ After fire $)$

Table 5: Comparative study of densities of herb species across the stand position before fire and after fire.

\section{Correlation Matrix}

The correlation matrix of different parameters with study sites (Before and after fire) is indicated in Table 6. Density (-0.988) and biomass (-0.977) showed strong negative correlation with study sites i.e. density and biomass of herbs decreased after fire in the studied site. Biomass (0.953) showed positive correlation with density i.e. biomass increased with increasing density. Diversity showed least significance with site and position. 


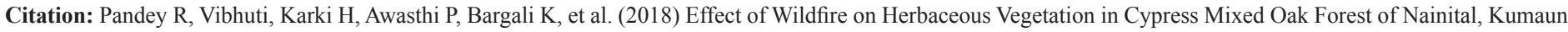
Himalaya, India. Curr Trends Forest Res: CTFR-121. DOI: 10.29011/2638-0013. 100021

\begin{tabular}{|c|c|c|c|c|c|}
\hline & Site & Position & D & H & B \\
\hline Site & 1 & 0.000 & $-0.988^{* *}$ & -0.418 & $-0.977^{* *}$ \\
\hline Position & & 1 & -0.109 & 0.373 & 0.067 \\
\hline D & & & 1 & 0.462 & 1 \\
\hline H & & & & $0.953^{* *}$ & 0.313 \\
\hline B & & & & 1 \\
\hline
\end{tabular}

Table 6: Pearson Correlation matrix for the study site before and after fire.

\begin{tabular}{|c|c|c|c|c|c|c|c|}
\hline \multirow[t]{2}{*}{ Herb species } & \multirow[t]{2}{*}{ Family } & \multicolumn{3}{|c|}{ Present study(After fire) } & \multicolumn{3}{|c|}{ Karki et al. 2016 (Before fire) } \\
\hline & & $\begin{array}{l}\text { Hill } \\
\text { base }\end{array}$ & $\begin{array}{c}\text { Hill } \\
\text { slope }\end{array}$ & $\begin{array}{l}\text { Hill } \\
\text { top }\end{array}$ & $\begin{array}{c}\text { Hill } \\
\text { base }\end{array}$ & $\begin{array}{c}\text { Hill } \\
\text { slope }\end{array}$ & Hill top \\
\hline Achyranthes bidentata Blume & Amaranthaceae & + & + & + & + & + & + \\
\hline Ageratum conyzoides L. & Asteraceae & - & + & - & - & - & - \\
\hline Ainsliaea aptera DC. & Asteraceae & - & + & - & + & + & + \\
\hline Artemisia annua Linn. & Asteraceae & + & - & + & + & - & - \\
\hline Arthraxon lanceolatus. Thunb & Poaceae & - & - & - & + & + & + \\
\hline Bidens alba (L) DC. & Asteraceae & + & - & - & - & - & - \\
\hline Cardamine amara $\mathrm{L}$. & Brassicaceae & + & - & - & - & - & - \\
\hline Carex hirta L. & Cyperaceae & - & + & - & - & - & - \\
\hline Cassia occidentalis L. & Fabaceae & - & - & + & - & - & - \\
\hline Clematis buchaniana DC. & Ranunculaceae & - & + & + & + & - & + \\
\hline Conyza agerotoides DC. & Asteraceae & + & - & - & - & - & - \\
\hline Craniotome versicolor. Reichb. & Lamiaceae & - & - & + & + & + & - \\
\hline Crysopogon zizanioides (L.)Roberty & Poaceae & - & - & + & - & - & - \\
\hline Cyanotis axillaris (L.)D.Don ex Sweet & Commelinaceae & - & - & + & - & - & - \\
\hline Cynodon dactylon (L.)Pers. & Poaceae & + & - & + & + & + & - \\
\hline Cynoglossum officinale $\mathrm{L}$. & Boraginaceae & - & - & + & - & - & - \\
\hline Cyperus rotundus $\mathrm{L}$. & Cyperaceae & - & - & - & - & + & + \\
\hline Dicliptera bupleuroides Nees & Acanthaceae & + & + & - & - & + & + \\
\hline Dioscorea deltoidea Kunth. & Dioscoreaceae & - & - & - & + & - & - \\
\hline Erigeron bellidiodes L. & Asteraceae & + & - & - & + & - & - \\
\hline Eupatorium adenophorum Spreng & Asteraceae & + & - & + & + & - & - \\
\hline Fragaria vesca Linn. & Rosaceae & + & - & + & + & - & - \\
\hline Gallium aparina $\mathrm{L}$. & Rubiaceae & - & - & + & + & - & - \\
\hline Gallium rotundifolium L. & Rubiaceae & - & - & - & - & + & + \\
\hline Gebera gossypina (Royle) Beauv. & Asteraceae & - & - & - & - & + & + \\
\hline Goldfussia dalhousianus Clarke. & Acanthaceae & - & - & - & + & + & + \\
\hline
\end{tabular}




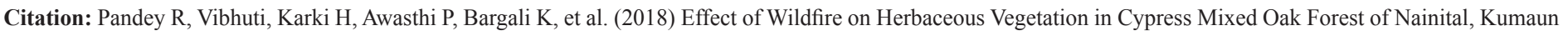
Himalaya, India. Curr Trends Forest Res: CTFR-121. DOI: 10.29011/2638-0013. 100021

\begin{tabular}{|c|c|c|c|c|c|c|c|}
\hline Hedychium spicatum BuchHam.ex J.E.Smith & Zingiberaceae & - & - & - & - & - & + \\
\hline Justicia simplex D. Don & Acanthaceae & - & + & - & - & - & - \\
\hline Malaxis acuminate D.Don.Roxb. & Orchidaceae & - & - & - & + & - & - \\
\hline Micromeria biflora (Don)Benth. & Lamiaceae & - & - & + & - & - & - \\
\hline Onychychium cryptogrammoides C. Chr & Cryptogrammaceae & - & - & - & - & + & - \\
\hline Parietaria officinalis L. & Urticaceae & - & - & + & - & - & - \\
\hline Paspalum conjugatum Hochst.ex Steud. & Poaceae & - & - & + & - & - & - \\
\hline Poa annua L. & Poaceae & - & + & - & - & - & - \\
\hline Randia densiflora $\mathrm{L}$. & Rubiaceae & - & + & - & - & - & - \\
\hline Roylea cinerea (D. Don) Baill. & Lamiaceae & + & - & - & - & - & - \\
\hline Salvia officinalis L. & Lamiaceae & + & - & - & - & - & - \\
\hline Seigesbeckia orientalis L. & Asteraceae & + & - & - & - & - & - \\
\hline Selaginella spp & Selaginellaceae & - & + & + & + & + & + \\
\hline Stellaria media (L)Vill & Caryophyllaceae & - & - & + & - & + & - \\
\hline Strobilanthes callosa (Nees) Bremek & Acanthaceae & - & + & - & - & - & - \\
\hline Thalictrum foliolosum DC. & Ranunculaceae & - & + & - & - & - & + \\
\hline Tragopogon gracilis D. Don & Asteraceae & - & - & - & - & - & + \\
\hline Verbascum thapsus L. & Scrophulariaceae & + & - & + & - & - & - \\
\hline Viola serpens Wall. & Violaceae & - & - & - & - & - & + \\
\hline Vitis himalayana Brandis & Vitaceae & - & + & - & + & + & + \\
\hline Voila canescens Wall. ex Roxb. & Voilaceae & - & - & - & + & - & - \\
\hline
\end{tabular}

Table 7: A list of herb species encountered in the studied area before and after fire $(+$ sign indicates the presence of species and -sign indicates its absence).

\section{Conclusion}

The present study is an attempt to investigate the role of fire in shaping ecosystem with emphasis on long and short term impact of fire and other biotic/ abiotic factors in combination with fire which cause biodiversity loss. The species composition and richness were significantly affected by fire but the magnitude of effects was generally small. Overall species composition became different in burned areas than in unburned areas. Certain burned areas also developed greater small-scale species richness than that of unburned areas. A group of perennial herbs, primarily grasses exhibited significant changes in their frequencies after fire that may result in long term changes in composition and richness.

From conservation prospective for successful rehabilitation of burnt sites a detailed study of all the vegetation layers and physico-chemical properties of soil could be helpful to conclude the effect of fire in vegetation and soil nutrient pool. Plantations could be established in accessible sites by using fast-growing native species in order to speed up the gap filling and carbon sequestration potential.

\section{Acknowledgement}

We are thankful to the Head, Department of Botany, DSB Campus for providing necessary lab facilities.

\section{References}

1. Rikhari HC, Adhikari BS, Rawat YS (1997) Woody species composition of temperate forests along an elevational gradient in Indian central Himalaya. J Trop for Sci 10: 197-211.

2. Singh JS, Singh SP (1987) Forest vegetation of Himalaya. Bot Rev 53: 80-192.

3. Bargali K, Joshi B, Bargali, SS, Singh SP (2014) Diversity within Oaks. Int Oaks 25: 57-70. 
Citation: Pandey R, Vibhuti, Karki H, Awasthi P, Bargali K, et al. (2018) Effect of Wildfire on Herbaceous Vegetation in Cypress Mixed Oak Forest of Nainital, Kumaun Himalaya, India. Curr Trends Forest Res: CTFR-121. DOI: 10.29011/2638-0013. 100021

4. Bargali K, Joshi B, Bargali SS, Singh SP (2015) Oaks and the Biodiversity They Sustain. Int Oaks 26: 65-76.

5. Mourya NR, Bargali K, Bargali SS (2018) Effect of Coriaria nepalensis Wall. colonization in a mixed conifer forest of Indian Central Himalaya. J For Res.

6. Shahni KC (1990) Gymnosperms of India and adjacent countries. Bishan Singh and Mahendra Pal Singh Dehradun, India.

7. Troup RS (1921) The Silviculture of Indian trees. Clarendon Press, Oxford 111: 1195.

8. Champion HG, Seth SK (1968) A Revised Survey of the Forest Types of India. Government of India Publication, New Delhi.

9. Baduni NP and Sharma CM (1996) Effect of aspect on the structure of some natural stands of Cupressus torulosa in Himalayan moist temperate forest. Proc of Indian Natl Sci Acad 62: 345-352.

10. Bargali SS (1994) Forest floor development in Eucalyptus tereticornis Sm. plantations in a part of Central Himalayan tarai belt. Range Manag and Agrofor 15: 61-68.

11. Gilliam FS, Roberts MR (2003) The herbaceous layer in forests of eastern North America. New York: Oxford University Press 408.

12. Bargali SS, Bargali K (2000) Diversity and biomass of the under story vegetation in an age series of Eucalyptus tereticornis plantation. Int $\mathrm{J}$ Ecol Environ Sci 26: 173-181.

13. Augusto L, Dupouey JL, Ranger J (2003) Effects of tree species on understorey vegetation and environmental conditions in temperate forests. Ann for Sci 60: 823-831.

14. Whigham DF (2004) Ecology of woodland herbs in temperate deciduous forests. Annu Rev Ecol Evol 35: 583-621.

15. Hutchinson TF, Boerner REJ, Iverson LR, Sutherland S, Sutherland EK (1999) Landscape patterns of understory composition and richness across a moisture and nitrogen mineralization gradient in Ohio (U.S.A.) Quercus forests. Plant Ecol 144: 179-189.

16. Shahi C, Sharma A, Bargali K, Bargali SS (2014) Effect of disturbance gradient on forest floor biomass in natural forest ecosystems in and around Nainital. The Ecoscan 8: 163-168.

17. Small CJ, McCarthy BC (2002) Spatial and temporal variation in the response of understory vegetation to disturbance in a central Appalachian oak forest. J Torrey Bot Soc 129: 136-153.

18. Kittur B, Swamy SL, Bargali SS, Jhariya MK (2014) Wildland Fires and Moist Deciduous Forests of Chhattisgarh, India: Divergent Component Assessment. J for Res 25: 857-866.

19. Show SB, Clarke B (1978) Forest Fire Control; FAO 104-105.

20. Gilliam FS (1988) Interactions of fire with nutrients in the herbaceous layer of a nutrient-poor Coital Plain forest. Bull. Torrey Bot Club 115: 265-271.

21. Kruger EL, Reich PB (1997) Responses of hardwood regeneration to fire in mesic forest openings. Post-fire community dynamics. Can J for Res 27: 1822-1831.

22. Ryan KC (2002) Dynamic interactions between forest structure and fire behavior in boreal ecosystems. Silva Fenn 36: 13-39.
23. McGee GG, Leopold DJ, Nyland RD (1995) Understory response to springtime prescribed fire in two New York transition oak forests. For Ecol and Manag 1: 149-168.

24. Ducey MJ, Moser WK, Ashton PMS (1996) Effect of fire intensity on understory composition and diversity in a Kalmia-dominated oak forest, New England, U.S.A. Vegetation 123: 81-90.

25. Arthur MA, Paratley RD, Blankenship BA (1998) Single and repeated fires affect survival and regeneration of woody and herbaceous species in an oak-pine forest. J Torrey Bot Soc 125: 225-236.

26. Franklin SB, Robertson FA, Pralish JS (2003) Prescribed burning effects on upland Quercus forest structure and function. For Ecol and Manag 184: 315-335

27. Osmaston AE (1980) A forest flora for Kumaon Govt. press, Allahabad, United province.

28. Gupta RK (1968) Flora Nainitalensis. Nav Yug Traders, New Delhi.

29. Gaur RD (1999) Flora of the District Garhwal North West Himalaya with ethnobotanical notes. Transmedia Publications, Srinagar Garhwal.

30. Pande HC, Pande PC (2003) An Illustrated Fern Flora of the Kumaun Himalaya. Vo. I and II. Dehradun.

31. Saxena AK, Singh JS (1982) A phytosociological analysis of woody species in forest communities of a part of Kumaun Himalaya. Vegetation 50: 3-22.

32. Curtis JT, Mcintosh RP (1950) The interrelations of certain analytic and synthetic phytosociological characters. Ecology 31: 438-455.

33. Curtis JT (1959) The vegetation of Wisconsin. An ordination of plant community University Wisconsin Press, Madison, Wisconsin: 657

34. Curtis JT, Cottam G (1956) Plant ecology work book. Laboratory field reference manual. Burgess Publication Co., Minnesota: 193.

35. Shannon CE, Wiener W (1963) The Mathematical Theory of Communication. University of Illinois Press, Urbana.

36. Simpson EH (1949) Measurement of diversity. Nature 168: 688.

37. Whittaker RH (1960) Vegetation of the Siskiyou mountains, Oregon and California. Ecol Monogr 30: 279-338.

38. Pielou EC (1966) The measurement of diversity in different types of biological collections. J Theor Biol 13: 131-144.

39. Mueller-Dombois D, Ellenburg H (1974) Aims and methods of vegetation Ecology. Jhon Wiley and Sons. Inc.

40. Karki H, Rana P, Bargali K, Bargali SS, Rawat YS (2016) Effect of Biotic disturbances on herbaceous vegetation in Cypress Mixed Oak Forests of Central Himalaya, India. Curr World Environ 11: 413-422.

41. Pande R, Bargali K, Pande N (2014) Impacts of disturbance on the population structure and regeneration status of tree species in a Central Himalayan mixed-oak forest India. Taiwan J for Sci 29: 179-192.

42. Sheil D (1999) Tropical forest diversity environmental change and species augmentation after the intermediate disturbance hypothesis. J Veg Sci 10: 851-860.

43. Swan FR (1970) Post-fire response of four plant communities in southcentral New York state. Ecology 51: 1074-1082. 
Citation: Pandey R, Vibhuti, Karki H, Awasthi P, Bargali K, et al. (2018) Effect of Wildfire on Herbaceous Vegetation in Cypress Mixed Oak Forest of Nainital, Kumaun Himalaya, India. Curr Trends Forest Res: CTFR-121. DOI: 10.29011/2638-0013. 100021

44. Nuzzo VA, McClain W, Strole T (1996) Fire impact on groundlayer flora in a sand forest 1990-1994. Am Midl Nat 136: 207-221.

45. Elliot KJ, Hendrick L, Major AE, Vose JM, Swank WT (1999) Vegetation dynamics after prescribed fire in the southern Appalachians. For Ecol Manag 114: 199-213.

46. Kuddes-Fischer LM, Arthur MA (2002) Response of understory vegetation and tree regeneration to a single prescribed fire in oak-pine forests. Nat Area J 22: 43-52.

47. Facelli JM, Pickett STA (1991) Plant litter: its dynamics and effects on plant community structure. Bot Rev 57: 1-32.
48. Gilliam FS, Christensen NL (1986) Herb-layer response to burning in pine flatwoods of the lower Coastal Plain of South Carolina. Bull Torrey Bot Club 113: 42-45.

49. Auchmoody LR (1979) Nitrogen fertilization stimulates of dormant pin cherry seed. Can J for Res 9: 514-516.

50. Odum EP (1971) Fundamentals of Ecology (III edition) W.B. Saunders Co., Philadelphia, U.S.A. 574. 